of C. trachomatis infectious cycle. Inhibiting lipid peroxidation using ferrostatin-1 and liproxstatin-1 not only abolished C. trachomatis-induced cell death, but also blocked C. trachomatis egress. Three C. trachomatis biovars were found to disseminate via ferroptosis, indicating that it is a conserved process. Conclusion C. trachomatis hijacks host cell machinery of ferroptosis in the late stage of infection for dissemination.

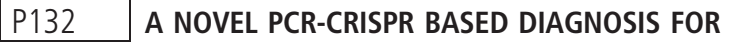 TREPONEMA PALLIDUM DETECTION, GENOTYPING, AND DRUG-RESISTANCE MUTATION IDENTIFICATION IN REAL-TIME}

W Chen*, H Luo, Z Mai, Y Jiang, Z Hu, L Zeng, H Zheng. Dermatology Hospital, Southern Medical University, Guangzhou, China

\subsection{6/sextrans-2021-sti.246}

Background The sexually transmitted disease syphilis caused by Treponema pallidum subsp. pallidum (TPA), is resurging worldwide, particularly in developing countries. Nucleic acid amplification tests (NAATs) possess highly sensitive and specifical capabilities, which have been largely utilized for pathogen detection, e. g. virus, bacteria, and fungus. However, the traditional NAATs applied to diagnose TPA are insufficiently sensitive.

Methods We established a novel PCR-CRISPR based syphilis molecular diagnosis with multiple excellent utilities.

Results The new approach retains the advantage of the high specificity of NAATs and allows detecting TPA with low concentrations at the single molecular level, which is more sensitive than the classical PCR assay. We also adapt this method to analyze the TPA by Nichols-/SS14-like cluster genotyping and macrolide resistance-associated mutations detection in realtime, providing a powerful tool for improving syphilis surveillance and clinical decisions/treatment selections. We further applied these assays to evaluate a panel of 42 TPA strains circulating in Southern China and find that the prevalent TPA strains consist of $83.33 \%$ SS14- and $16.67 \%$ Nichols-like TPA strains, with $97.67 \%$ of strains harboring $23 \mathrm{~s}$ rRNA mutations (A2058G or A2059G).

Conclusion We developed a novel PCR-CRISPR-based syphilis molecular assay, which exhibits robust capabilities and prospects in the diagnosis and molecular epidemiology of TPA.

\section{P135 HIGH PREVALENCE OF CURABLE SEXUALLY TRANSMITTED INFECTIONS AMONG 14-19 YEAR OLD ADOLESCENTS AT RISK OF HIV INFECTION IN KAMPALA, UGANDA}

${ }^{1} \mathrm{Y}$ Mayanja*, ${ }^{1} \mathrm{~J}$ Lunkuse, ${ }^{1} \mathrm{H}$ Kalungi, ${ }^{2} \mathrm{~W}$ Senyonga, ${ }^{1} \mathrm{O}$ Kamacooko, ${ }^{1} \mathrm{E}$ Ruzagira. ${ }^{1} \mathrm{MRC} /$ UVRI and LSHTM Uganda Research Unit, Entebbe, Uganda; ${ }^{2}$ Ministry of Health, Kampala, Uganda

\subsection{6/sextrans-2021-sti.247}

Background Sexually transmitted infections (STIs) are of public health significance; they have adverse reproductive health consequences and yet are preventable and curable. We studied STI prevalence and associated factors among adolescents in Kampala, Uganda.

Methods We conducted a cross-sectional study among 14-19year-old high-risk adolescents from March 2019 to March 2020. An interviewer-administered questionnaire was used to collect socio-demographic and behaviour data. STI tests were performed on urine using geneXpert for Chlamydia trachomatis (CT) and Neisseria gonorrhoeae (NG), and serum using the Rapid plasma reagin/Treponema Pallidum Particle Agglutination Assay for syphilis. Outcomes were positive tests for $\mathrm{CT}, \mathrm{NG}$, and/or active syphilis (titre $\geq 1: 8$ ). Statistical analysis was conducted using logistic regression.

Results We enrolled 490 volunteers with a median age of 18 years (Interquartile range 17-18 years). Most were female $(60.6 \%), \geq 18$ years $(50.8 \%)$, had only primary school education $(91.0 \%)$ and had their sexual debut at $<15$ years (48.4\%). Females reported a higher prevalence of paid sex than males both at the last sexual encounter $(96.4 \%$ vs $3.6 \%, \mathrm{p}<0.001)$ and in the past 3 months $(94.8 \%$ vs. $5.2 \%$, $\mathrm{p}<0.001) ; 128(26.1 \%)$ individuals reported change of residence in the past year with the frequency being higher in females than in males $(82 \%$ vs $18 \%, \mathrm{p}<0.001)$. STI prevalence was 39\% [CT (21.4\%), NG (5.9\%), active syphilis (1.4\%), multiple infections (10.3\%)] and higher among females than males $(75 \%$ vs $25 \% ; \mathrm{p}<0.001)$. Prevalent STIs were associated with paid sex at the last encounter (aOR 1.78 ; 95\% CI 1.02-3.16) and change of residence in the past year. The effect of changing residence was modified by sex; being significant for female (aOR 2.17; 95\% CI 1.293.67 ) but not male (aOR $0.26 ; 95 \%$ CI $0.33-2.00$ ) participants.

Conclusions Curable STIs are highly prevalent in this adolescent population. Interventions should increase access to STI prevention and treatment in sex work hotspots.

\section{P137 VIRAL HEPATITIS PREVALENCE AMONG MEN WHO HAVE SEX WITH MEN RECRUITED IN AN HIV PREP DEMONSTRATION STUDY IN COTONOU, BENIN}

\begin{abstract}
1,2,3 $\mathrm{L}$ Behanzin*, ${ }^{2,3} \mathrm{~F}$ Guédou, ${ }^{2} \mathrm{M}$ Goma, ${ }^{2} \mathrm{M}$ Olodo, ${ }^{2} \mathrm{M}$ Aza-Gnandji, ${ }^{2} \mathrm{~A}$ Dossouvo, ${ }^{2} \mathrm{~F}$ Kintin, ${ }^{4} \mathrm{~A}$ Akpaka, ${ }^{5} \mathrm{E}$ Chagas, ${ }^{6} \mathrm{~S}$ Ogoundélé, ${ }^{6} \mathrm{C}$ Ahouada, ${ }^{7,8,9} \mathrm{~F}$ Gangbo, ${ }^{8,9} \mathrm{M}$ Zannou, $3,10,11$ M Alary. ${ }^{1}$ Ecole Nationale de Formation des Techniciens Supérieurs en Santé Publique et en Surveillance Epidémiologique (ENATSE), Université de Parakou, Parakou, Bénin; ${ }^{2}$ Dispensaire IST, Centre de santé communal de Cotonou 1, Cotonou, Bénin; ${ }^{3}$ Axe Santé des populations et pratiques optimales en santé, Centre de recherche du CHU de Québec Université Laval, Québec, Canada; ${ }^{4}$ BENIN SYNERGIES PLUS (BeSyP), Cotonou, Bénin; ${ }^{5}$ RESEAU SIDA BENIN (RSB), Cotonou, Bénin; ${ }^{6}$ Plan International Bénin, Cotonou, Bénin; ${ }^{7}$ Programme Santé de Lutte contre le Sida (PSLS), Cotonou, Bénin; ${ }^{8}$ Faculté des sciences de la santé, Université d'Abomey-Calavi, Cotonou, Bénin; ${ }^{9}$ Centre national hospitalier universitaire HMK de Cotonou, Cotonou, Bénin; ${ }^{10}$ Département de médecine sociale et préventive, Université Laval, Québec, Québec, Canada; ${ }^{11}$ Institut national de santé publique du Québec, Québec, Canada
\end{abstract}

\subsection{6/sextrans-2021-sti.248}

Background Men who have sex with men (MSM) are disproportionally affected by hepatitis worldwide despite the availability of safe and effective vaccines for hepatitis A and B. The aim of this analysis was to estimate the prevalence and risk factors for current and lifetime hepatitis B, and HCV antibodies among MSM in Cotonou.

Methods A two-stage cluster sampling procedure was used to recruit 204 MSM in the community-based PrEP demonstration study. First, 7 out of the 13 boroughs in Cotonou were selected using a probability proportional to size method and a fixed number of MSM were then randomly selected from each borough using a random route sampling procedure. A rapid immuno-chromatographic test was used for detecting HBV surface antigen (HBsAg, active 